\title{
CENTERS OF CURVATURES OF A VECTOR FIELD
}

\author{
T. K. PAN
}

1. Introduction. Let $v$ denote a unit vector field on a surface in three dimensional euclidean space. With respect to a curve $C$ on the surface, $v$ has several curvatures, of which the angular spread or associate curvature was studied by Graustein $[2]^{1}$ and the normal curvature by the author [3]. In this paper, definitions of the following centers of curvatures of $v$ with respect to $C$-center of normal curvature, center of angular spread, and center of absolute curvature-are given to include those of a curve on a surface as a special case. Properties of these centers of various curvatures of $v$ with respect to $C$ are then investigated. Most of the theorems for centers of curvatures of a curve on a surface are generalized.

The notation of Eisenhart [1] will be used for the most part.

2. Definitions. Let a surface $S$ in three dimensional euclidean space be defined with reference to a rectangular cartesian coordinate system by $^{2} x^{i}=x^{i}\left(u^{1}, u^{2}\right)$ and be of class not less than 2. Let $g_{\alpha \beta}$ and $d_{\alpha \beta}$ be, respectively, the first and second fundamental tensors of $S$ such that $g=\left|g_{\alpha \beta}\right|>0$ and $d=\left|d_{\alpha \beta}\right| \neq 0$.

Associate to each point of $S$ an arbitrary but fixed unit vector with components $v^{i}=x^{i},{ }_{\alpha} p^{\alpha}$; thus $g_{\alpha \beta} p^{\alpha} p^{\beta}=1$. These vectors on $S$ form a unit vector field and will be denoted by $v$ or $p$ at appropriate places. We assume $v$ to be of class not less than 1 .

Let $C$ be a curve on $S$, which is defined by $u^{\alpha}=u^{\alpha}(s), s$ being its arc length. Let $C$ be of class not less than 2. Let $P\left(x^{i}\right)$ be a generic point of $C$ and $X^{i}$ the components of the unit vector normal to $S$ at $P$. Denote by $q^{\alpha}$ the unit vector in $S$, which makes a right angle with $p^{\alpha}$ at $P[1$, p. 132, p. 187]. Then the derived vector of $v$ with respect to $C$ at $P$ is defined by

$$
\frac{d v^{i}}{d s}={ }_{v} K w^{i}={ }_{v} K_{0} q^{\alpha} x^{i},{ }_{\alpha}+{ }_{v} K_{n} X^{i}
$$

where

Presented to the Society, November 27, 1954; received by the editors November 4, 1954, and, in revised form, April 22, 1955.

${ }^{1}$ Numbers in brackets refer to the references at the end of this paper.

2 All Latin indices in this paper run from 1 to 3 and all Greek indices from 1 to 2 . 


$$
{ }_{v} K_{o} q^{\alpha}=p^{\alpha}, \frac{d u^{\gamma}}{d s}, \quad{ }_{v} K_{n}=d_{\delta \gamma} p^{\delta} \frac{d u^{\gamma}}{d s}
$$

and where $w^{i}$ are the components of a unit vector taken in the same sense of $d v^{i} / d s$ so that ${ }_{v} K$ is always positive.

The vector defined by the left-hand member of (2.1) is called the absolute curvature vector of $v$ with respect to $C$. The two terms in the right-hand member of (2.1) are, respectively, the tangential and normal components of the absolute curvature vector of $v$ with respect to $C$; the vectors defined by them are called the angular spread vector of $v$ with respect to $C$ and the normal curvature vector of $v$ with respect to $C$, respectively. The magnitudes of all these three vectors, which are denoted by ${ }_{v} K,{ }_{v} K_{g}$, and ${ }_{v} K_{n}$, are called, respectively, the absolute curvature of $v$ with respect to $C$, the angular spread of $v$ with respect to $C$, and the normal curvature of $v$ with respect to $C[2$, p. $559 ; 3$, pp. 956-961].

The direction, with respect to which the normal curvature of $v$ at $P$ has its extreme value, is known as the principal direction of $v$ at $P$, and the corresponding normal curvature is called the principal curvature of $v$ at $P$. A curve on $S$, whose direction at each and every point is a principal direction of $v$, is called a line of curvature of $v$. It is defined by

$$
\epsilon^{\alpha \beta} g_{\alpha \gamma} d_{\beta \delta} p^{\delta} d u^{\gamma}=0 .
$$

The direction, with respect to which the normal curvature of $v$ at $P$ is zero, is called the asymptotic direction of $v$ at $P$. A curve on $S$, whose direction at each and every point is an asymptotic direction of $v$, is called an asymptotic line of $v$. It is defined by

$$
d_{\delta \gamma} p^{\delta} d u^{\gamma}=0 .
$$

The curves of $v$ and the asymptotic lines of $v$ form a conjugate net on $S$.

A curve on $S$, with respect to which the vectors of $v$ are parallel in the sense of Levi-Civita, is called an indicatrix of $v$. It is defined by

$$
p^{\alpha}, \gamma d u^{\gamma}=0 \text {. }
$$

Let the unit vector $v$ in $S$ at $P$ undergo a local displacement to $v_{1}$ from $P$ with respect to $C$ and a parallel displacement to $v_{2}$ from $P$ with respect to $C$. Let $\phi$ be the angle which $v_{2}$ makes with $v_{1}$. It is found that

$$
\frac{d \phi}{d s}=-{ }_{v} K_{\sigma}
$$


Let $\omega$ be the angle between $X^{i}$ and $w^{i}$. It is found that

$$
{ }_{v} K_{n}={ }_{v} K \cos \omega
$$

which is analogous to Meusnier's theorem. If ${ }_{v} K \neq 0,{ }^{\circ} K_{n}$ is positive or negative according as $0 \leqq \omega<\pi / 2$ or $\pi \geqq \omega>\pi / 2$.

Let the quantities ${ }_{\bullet} r_{,}{ }_{0} r_{\theta}$, and ${ }_{\bullet} r_{n}$ be defined as follows:

$$
{ }_{v} r=\frac{1}{{ }_{v} K}, \quad{ }_{v} r_{0}=\frac{1}{{ }_{v} K_{0}}, \quad{ }_{v} r_{n}=\frac{1}{{ }_{v} K_{n}} .
$$

The first quantity, ${ } r$, is always positive since ${ }_{v} K$ is defined as positive. The other two quantities, ${ }_{\bullet} r_{\theta}$ and ${ }_{\bullet} r_{n}$, may be positive or negative.

Let $\theta$ denote the angle between $C$ and $v$ at $P$. At $P$ of $C$, the point on the absolute curvature vector of $v$ with respect to $C$ at the distance or $\cos \theta$ from $P$ is defined as the center of absolute curvature for $P$ of $v$ with respect to $C$, the point on the angular spread vector of $v$ at the distance ${ }_{\bullet} r_{\theta} \cos \theta$ from $P$ is defined as the center of angular spread for $P$ of $v$ with respect to $C$, and the point on the surface normal at the distance ${ }_{v} r_{n} \cos \theta$ from $P$ is defined as the center of normal curvature for $P$ of $v$ with respect to $C$.

3. Properties. It is shown $\left[3\right.$, p. 956] that ${ }_{v} K_{n}$ is zero with respect to any curve in $S$ at $P$ if and only if $d=0$ at $P$. Since $S$ is assumed to be a surface with $d \neq 0$, we have by (2.2) and (2.4) that ${ }_{0} K_{n}$ is zero with respect to $C$ at $P$ if and only if $C$ is in the direction conjugate to $v$ at $P$.

When $K_{n}$ is different from zero, equation (2.7) can be cast into the form

$$
{ }_{v} r={ }_{v} r_{n} \cos \omega
$$

which implies geometrically that the center of absolute curvature for $P$ of $v$ with respect to $C$, which is not conjugate to $v$ at $P$, is the orthogonal projection of the center of normal curvature for $P$ of $v$ with respect to $C$ on the absolute curvature vector of $v$ with respect to $C$ at $P$.

The following relation between ${ }_{v} K_{0}$ and ${ }_{\bullet} K$ can be derived readily

$$
{ }_{{ }} K_{\theta}=e_{\nu} K \sin \omega
$$

where $e$ is +1 or -1 as the case may be. The quantity ${ }_{0} K_{0}$ is zero with respect to any curve on $S$ if and only if $v$ is a field of absolutely parallel unit vectors in $S$, that is, $S$ is isometric with the plane [1, p. 198]. This implies $d=0$. Since $S$ is assumed to be a surface with $d \neq 0$, we have from (2.2) and (2.5) that ${ }^{\circ} K_{o}$ is zero with respect to $C$ at $P$ if and only if $C$ is in the direction of the indicatrix at $v$ at $P$. 
When ${ }_{v} K_{g}$ is different from zero, equation (3.2) can be cast into the form

$$
{ }_{v} r=e{ }_{v} r_{g} \sin \omega,
$$

which implies geometrically that the center of absolute curvature for $P$ of $v$ with respect to $C$, which is not tangent to the indicatrix of $v$ at $P$, is the orthogonal projection of the center of angular spread for $P$ of $v$ with respect to $C$ on the absolute curvature vector of $v$ with respect to $C$ at $P$.

Considering equations (3.1) and (3.3) simultaneously, we have

THEOREM 3.1. With respect to $C$ at $P$ which is neither in the direction conjugate to $v$ at $P$ nor in the direction of the indicatrix of $v$ at $P$, the three centers of curvatures for $P$ of $v$-center of absolute curvature, center of angular spread, and center of normal curvature-are collinear.

Since the unit angular spread vector $q^{\alpha}$ of $v$ with respect to $C$ is orthogonal to $p^{\alpha}$, multiplication of the first equation of (2.2) by $\epsilon_{\beta \alpha} p^{\beta}$ and summation over $\alpha$ give

$$
{ }_{v} K_{0}=\frac{\epsilon_{\rho \alpha} p^{\rho}\left(p^{\alpha},{ }_{11}+p^{\alpha},{ }_{2} \lambda\right)}{\left(g_{11}+2 g_{12} \lambda+g_{22} \lambda^{2}\right)^{1 / 2}},
$$

where $\lambda$ denotes $d u^{2} / d u^{1}$. The extreme value of the angular spread of $v$ with respect to $C$ as $C$ varies is called the principal angular spread of $v$. It is given by the direction $\lambda$ for which $d\left({ }_{v} K_{0}\right) / d \lambda=0$, that is, the direction defined by

$$
\lambda=\frac{\epsilon^{\gamma \delta} g_{\delta 1} \epsilon_{\rho \alpha \alpha} p^{\rho} p^{\alpha}, \gamma}{\epsilon^{\omega \tau} g_{\omega 2} \epsilon_{\mu \beta} p^{\mu} p^{\beta},} .
$$

Such direction is called the direction of angular spread of $v$ or, simply, the angular direction of $v$. A curve on $S$, whose direction at each and every point is an angular direction of $v$, is called a line of angular spread of $v$. In terms of $d u^{\alpha}$, the angular direction of $v$ at $P$ and the line of angular spread of $v$ at $P$ are defined by

$$
g^{\tau \gamma} \epsilon_{\sigma \tau} \epsilon_{\rho \alpha} p^{\rho} p^{\alpha}, \gamma d u^{\sigma}=0 .
$$

From (3.5), we find with the aid of an elementary property of proportion

$$
\frac{d u^{1}}{d u^{2}}=\frac{g^{1 \gamma} \epsilon_{\rho \alpha} p^{\rho} p^{\alpha}, \gamma}{g^{2 \tau} \epsilon_{\beta \sigma} p^{\beta} p^{\sigma}{ }_{, \tau}}=\frac{g^{1 \gamma} p^{\omega}, \gamma}{g^{2 \gamma} p^{\omega}{ }_{, \gamma}} .
$$

Hence the angular direction of $v$ at $P$ and the line of angular spread of $v$ at $P$ can be equally defined by 


$$
g^{\tau \gamma} \epsilon_{\sigma \tau} p^{\alpha}{ }_{, \gamma} d u^{\sigma}=0 .
$$

From (2.5) we find the direction of the indicatrix of $v$ at $P$ is $d u^{\gamma}$ $=\epsilon^{\gamma \beta} p^{\alpha},{ }_{\beta}$. Hence the orthogonal trajectories of the family of indicatrices of $v$ are defined by

$$
g_{\gamma \delta} \epsilon^{\gamma \beta} p^{\alpha}{ }_{, \beta} d u^{\delta}=0
$$

which is evidently equivalent to (3.6) since $g^{\alpha \delta} \epsilon_{\beta \delta}=g_{\gamma \beta} \epsilon^{\gamma \alpha}$. Hence we have

THEOREM 3.2. The angular spread of $v$ at $P$ has an extreme value with respect to the line of angular spread of $v$ at $P$ and is equal to zero with respect to the indicatrix of $v$ at $P$. The family of lines of angular spread of $v$ and the family of indicatrices of $v$ form an orthogonal net on $S$.

The centers determined by the reciprocals of the principal curvature of $v$ and the principal angular spread of $v$ are called the principal center of normal curvature of $v$ and the principal center of angular spread of $v$, respectively. By (2.6) the angular spread of $v$ with respect to $C$ is evidently a measure of the deviation of $v$ from parallelism with respect to $C$. Hence any vector field in $S$ which makes a constant angle with $v$ at every point of $S$ will have equal angular spread with respect to $C$. The totality of such vector fields is known as a pencil of vector fields. Thus we have

THEOREM 3.3. The center of normal curvature for $P$ of $v$ with respect to $C$ moves away from the principal center of normal curvature for $P$ of $v$ to infinity as the direction of $C$ varies from the principal direction of $v$ at $P$ to the direction conjugate to $v$ at $P$. The center of angular spread for $P$ of $v$ moves away from the principal center of angular spread for $P$ of $v$ to infinity as the direction of $C$ varies from the angular direction of $v$ at $P$ to the direction of the indicatrix of $v$ at $P$. The locus of the centers of angular spread for $P$ of a pencil of unit vector fields with respect to $C$ is a circle with center at $P$ on the tangent plane to $S$ at $P$. The circle has a smallest radius when $C$ is the line of angular spread of any field of the pencil at $P$.

Let $C_{0}$ be the line of curvature of $v$ at $P$ and $C_{1}, C_{2}$ two curves making equal angles with $C_{0}$ at $P$. Denote the unit tangent vectors at $P$ of $C_{0}, C_{1}$, and $C_{2}$ by $l^{\alpha}, m^{\alpha}$, and $n^{\alpha}$, respectively. By $(2.3)$ we have $p^{\alpha}$ and $l^{\alpha}$ satisfy the following relation:

$$
\epsilon^{\alpha \beta} g_{\alpha \gamma} d_{\beta \delta} p^{\delta} l \gamma=0,
$$

from which we obtain

$$
d_{\beta \delta} p^{\delta}=g_{\beta \gamma} l^{\gamma}
$$


Substitution of (3.7) into

$$
g_{\alpha \beta} l^{\alpha} m^{\beta}=g_{\gamma \delta} l^{\gamma} n^{\delta}
$$

gives

$$
d_{\alpha \beta} p^{\alpha} m^{\beta}=d_{\gamma \delta} p^{\gamma} n^{\delta}
$$

which yields immediately the following theorem.

THEOREM 3.4. If two curves make equal angles with the line of curvature of $v$ at $P$, the centers of normal curvature for $P$ of $v$ with respect to these two curves are coincident.

From the definition of angular spread vector of $v$ with respect to $C$, we find readily

$$
q^{\beta}=\epsilon^{\gamma \beta} g_{\alpha \gamma} p^{\alpha} .
$$

Equation (3.9) is said to define the field of unit angular spread vectors of $v$ or the orthogonal unit vector field of $v$. Substituting (3.9) for $p^{\delta}$ in (2.4), we obtain the equation of the asymptotic lines of the orthogonal unit vector field of $v$, which turns out to be equivalent to (2.3) of the lines of curvature of $v$. Thus, the line of curvature of $v$ at $P$ is the same as the asymptotic line at $P$ of the orthogonal unit vector field of $v$. This result is synthetically obvious because of the conjugate relation between the curves of $v$ and the asymptotic lines of $v$.

It is shown [5] that the center of angular spread for $P$ of $v$ with respect to $C$ is identical with the orthocenter of associate curvature of the unit angular spread vector of $v$ as defined in $\left[4\right.$, p. 152]..$^{3}$ It is also proved $[4$, p. 157] that the straight lines on the vectors of a unit vector field along an asymptotic line of the field form generators of a developable surface whose edge of regression is the locus of the orthocenters of associate curvature of the field along that symptotic line of the field. Consequently, the straight lines on the angular spread vectors of $v$ along a line of curvature of $v$ form a developable surface. Hence we have

THEOREM 3.5. The locus of center of angular spread of $v$ with respect to a line of curvature of $v$ is the edge of regression on the developable sur-

\footnotetext{
${ }^{3}$ This definition of orthocenter is a metric one and is given for a vector field $v$ with respect to any curve $C$. When $C$ is the asymptotic line of $v$ at $P$, the orthocenter of associate curvature for $P$ of $v$ along $C$ is Wilczynski's ray point of $C$ at $P$ as pointed out by the referee. This coincidence is not clear from Wilczynski's projective definition, but is evident from a theorem (E. P. Lane, $A$ treatise on projective differential geometry, University of Chicago Press, pp. 184-185). Thus a few theorems such as 3.1, 4.1, and 4.2 in the paper [4], which form part of a metric generalization of a metric concept, appear at the same time as metric interpretations of projective theorems.
} 
face formed by straight lines on the angular spread vectors of $v$ along that line of curvature of $v$.

The above theorem is a generalization of the theorem that the locus of the centers of geodesic curvature of a line of curvature of a surface is an evolute of the line of curvature.

\section{REFERENCES}

1. L. P. Eisenhart, An introduction to differential geometry with the use of the tensor calculus, Princeton University Press, 1947.

2. W. C. Graustein, Parallelism and equidistance in classical differential geometry, Trans. Amer. Math. Soc. vol. 34 (1932) pp. 557-593.

3. T. K. Pan, Normal curvature of a vector field, Amer. J. Math. vol. 75 (1952) pp. 955-966.

4. - Complementary surfaces for a vector field, Proc. Amer. Math. Soc. vol. 6 (1955) pp. 151-158.

5.,$- A$ generalized theorem of center of geodesic curvature, Amer. Math. Monthly vol. 62 (1955) pp. 717-718.

UNIVERSITY OF OKIAHOMA 